\title{
Preparation and Characterization of Pineapple Leaf Nanocellulose by High Pressure Homogenization
}

\author{
Tiao-Kun FU ${ }^{1, a}$, Ji-Hua LI ${ }^{1, b,{ }^{*}}$, Xiao-Yi WEI ${ }^{1, \mathrm{c}}$, Fei WANG ${ }^{1, \mathrm{~d}}$, Li-Hong CUI ${ }^{1, \mathrm{e}}$ and \\ Yi-Hong WANG ${ }^{2, f}$
}

\author{
1 Key Laboratory of Tropical Crop Products Processing of Ministry of Agriculture, Agriculture \\ Products Processing Research Institute, Chinese Academy of Tropical Agricultural Sciences, China \\ 2 Food Science and Technology Department, Huazhong Agricultural University, China \\ aloveflyfree@126.com, ${ }^{\text {b }}$ foodpaper@126.com, 'weixywei@163.com, \\ drain-wf@163.com, ${ }^{\mathrm{e}} 21608212 @ q q . c o m,{ }^{\mathrm{f}} 363373150 @ q q . c o m$,
}

Keywords: Pineapple leaf, Ionic liquid, High pressure homogenization, Nanocellulose.

\begin{abstract}
Nanocellulose was extracted from pineapple leaf using high pressure homogenization $(\mathrm{HPH})$ in a liquid, homogeneous state. After optimizing conditions, nanocellulose was obtained at $100 \mathrm{MPa}$ for 45 cycles with the diameter of $4-10 \mathrm{~nm}$. The nanocellulose was cellulose II, with the crystallization index reducing from $69.68 \%$ to $34.25 \%$. The specific surface area of nanocellulose, Water-Holding Capacity and Oil-Holding Capacity were increased after high pressure homogeneous treatment. The new process can effectively open up a new direction for high-added-value applications of pineapple leaf.
\end{abstract}

\section{Introduction}

Pineapple leaf is a waste product of pineapple cultivation, which is found to be multicellular and lignocellulosic. The efficient utilization of this biomass for functional materials has attracted more attention around the world because of its high cellulose content with renewable characteristics. Recently, nanocellulose was used in material [1], electrical [2], medicine [3], chemical [4], food [5] and even superconductivity, due to its superior properties, such as high strength, low specific heat coefficient, large specific surface area, good water-holding capacity and good oil-holding capacity etc. In previous studies, nanocellulose was prepared through ball-milling process [6] and hydrolysis method $[7,8]$. However, there is still a big technical problem in industrial production: for example, mechanical method has the shortcoming of high energy consumption and low production efficiency [9]. Preparation of nanocellulose by hydrolysis method is low efficiency and caused serious pollution to the environment. Therefore, how to produce nanocellulose effectively, is still a problem to be resolved.

In this study, cellulose separation and refinement in liquid phase conditions, can reduce the cellulose chain between each other "sliding" resistance, and to exert certain physical force, make the fiber in a liquid, homogeneous state complete network structure collapse and the molecular chain of self-assembly [10,11]. pineapple leaf nanocellulose was prepared by cellulose liquid homogeneous nanometer technology, and was then characterized in physical and chemical properties, such as the particle size distribution in the water phase, specific surface area and porosity, swelling property, water-holding capacity, oil-holding capacity, etc. In order to later provides the theory basis for pineapple leaf fiber products nanotechnology applications.

\section{Experimental}

\section{Materials and Methods}

Pineapple leaf was obtained from zhanjiang plantations, collected by agricultural machinery research institute, Chinese academy of tropical agricultural sciences, China. The ionic liquid of 1-butyl-3-methylimidazolium chloride $([\mathrm{Bmim}] \mathrm{Cl})$ used in this study was synthesized in our previous 
study. All chemical reagents were of analytical grade, and purchased from Guangzhou Chemical Reagent Factory (Guangzhou, China).

Pretreatment of pineapple leaf and dissolution of cellulose: the original pineapple leaf was pretreated with sodium hydroxyl solution of $35 \mathrm{~g} / \mathrm{L}$ for $6 \mathrm{~h}$ at $95^{\circ} \mathrm{C}$ to remove the impurities.the cellulose was obtained with the ratio above $83.13 \mathrm{wt} \%$.Then certain cellulose (3wt\%) was dissolved in the synthesized ionic liquid at $150{ }^{\circ} \mathrm{C}$ by microwave heating with $400 \mathrm{~W}[11]$.

Preparation of nanocellulose by high pressure homogenizer: the cellulose/BmimCl solution was then passed through a high pressure homogenizer with a certain cycles at a certain pressure. Then five times as much as quality of boiled deionized water was added into the homogeneous solution, and stirred for $10 \mathrm{~min}$ on the magnetic stirrer to make the nanocellulose precipitation from homogeneous system. Afterwards repeatedly centrifugal washing, the supernatant fluid was clarificated by high-speed refrigerated centrifuge. Finally, the regenerated nanocellulose was dried in the vacuum freeze drier until reaching a constant weight[10].

\section{Analysis}

The structure properties of cellulose extracted from pineapple leaf before and after homogeneous isolation were characterized by transmission electron microscopy (TEM), infrared spectroscopy (FT IR), X-ray diffraction (XRD), thermo gravimetric analysis (TGA), and etc.

\section{Transmission Electron Microscopy (TEM) Analysis}

The morphology measurements of the pineapple leaf nanocellulose were characterized by transmission electron microscopy (TEM) (JEM-100, JEOL,Tokyo, Japan) operated at $100 \mathrm{keV}$.

\section{Infrared Spectroscopy (FT IR) Analysis}

Using $\mathrm{KBr}$ tabletting method to measure the infrared spectrum by the fourier transform infrared spectrometer (Spectrum GX-1, PerkinElmer, USA) in the range from 4000 to $400 \mathrm{~cm}^{-1}$.

\section{X-ray Diffraction (XRD) Analysis}

The crystal structure of the sample after homogeneous isolation was measured by X-ray Diffractometer (Rint-Ultima + , Rigaku, Japan). Technical parameters: $\mathrm{Cu}(\mathrm{K} \alpha$ wavelength $\lambda=0.154 \mathrm{~nm})$ radiation at $40 \mathrm{kV}$ and $20 \mathrm{~mA}$, at a speed of $0.04^{\circ} \mathrm{s}-1$ in the range $20-80^{\circ}$.

\section{Thermo Gravimetric Analysis (TGA)}

The thermo gravimetric curve was tested by thermo gravimetric analyzer (STA449C/4/G, Netzsch, Germany). Technical parameters: the static TGA mode, temperature range $25 \sim 800^{\circ} \mathrm{C}$, selects the $\mathrm{N}_{2}$ protection, heating rate $10^{\circ} \mathrm{C} / \mathrm{min}$.

\section{The Properties of Nanocellulose}

\section{Particle Size Measurements}

Particle sizes of nanocellulose under the condition of different homogeneous isolation were determined by the Nano-ZS (Malvern Instruments,UK), as the refractive index is 1.50 , the temperature is $25^{\circ} \mathrm{C}$. A little pineapple leaf nanocellulose was put into a beaker, then diluted in 100 $\mathrm{ml}$ deionized water with ultrasound for 3 min to make the solution dispersed evenly.

\section{The Specific Surface Area and Porosity}

The specific surface area and porosity of the samples were measured using the Brunauer-Emmett-Teller (BET) with five-point method under $77 \mathrm{~K}$ in $\mathrm{N}_{2}$ atmosphere (Surface area and porosity analyzer, ASAP2020-M,Micromeritics, USA).

\section{The Swelling Capacity}

$1.0000 \mathrm{~g}$ sample was immersed into a graduated cylinder at $25^{\circ} \mathrm{C}$ to observe the swelling behavior. After $24 \mathrm{~h}$, the expansion volume was measured until there was no further change in the volume. The degree of swelling ratio was measured by the following Eq.1[12]: 


$$
\mathrm{S}=\frac{V_{2}-V_{1}}{m}
$$

Where $\mathrm{S}$ is swelling capacity, $\mathrm{V}_{2}, \mathrm{~V}_{1}$ are the volume of solution with and without sample, respectively. $\mathrm{m}$ is the weight of sample.

\section{The Water-Holding Capacity}

$1.0000 \mathrm{~g}$ of sample was stirred in $10 \mathrm{ml}$ of deionized water for $24 \mathrm{~h}$ in a $50 \mathrm{ml}$ centrifuge tube at room temperature. After samples were centrifuged (4000g, $30 \mathrm{~min}$ ), the supernatant was transferred to a graduated cylinder of $10 \mathrm{~mL}$, where the volume was measured. Water-Holding Capacity was calculated as $10 \mathrm{ml}$ minus the volume of water in graduated cylinder[13].

\section{The Oil-Holding Capacity}

$1.0000 \mathrm{~g}$ of sample was stirred in $10 \mathrm{ml}$ of corn oil for $24 \mathrm{~h}$ in a $50 \mathrm{ml}$ centrifuge tube at room temperature. After samples were centrifuged (4000g, $30 \mathrm{~min}$ ), the supernatant was transferred to a graduated cylinder of $10 \mathrm{~mL}$, where the volume was measured., Oil-Holding Capacity was calculated as $10 \mathrm{ml}$ minus the volume of corn oil in graduated cylinder[13].

\section{Results and Discussion}

\section{Effects of HPH Pretreatment on Particle Sizes}

$3 \%(\mathrm{w} / \mathrm{w})$ pineapple leaf cellulose/BmimCl solution was passed through the high pressure homogenizer under $80 \mathrm{MPa}$ with different cycles at room temperature[14]. Fig. 1a shows the effects of different homogeneous cycles on particle size of pineapple leaf nanocellulose. With the increase of homogeneous cycles, the particle size of nanocellulose was decreased until 45 cycles and then increased again. It suggested that the network of pineapple leaf cellulose was broken with the increasing homogeneous cycles at first. But when the particle sizes reduced to a certain extent, the intermolecular attraction became stronger, and there were much stronger reunion between particles to obtain bigger particle sizes.

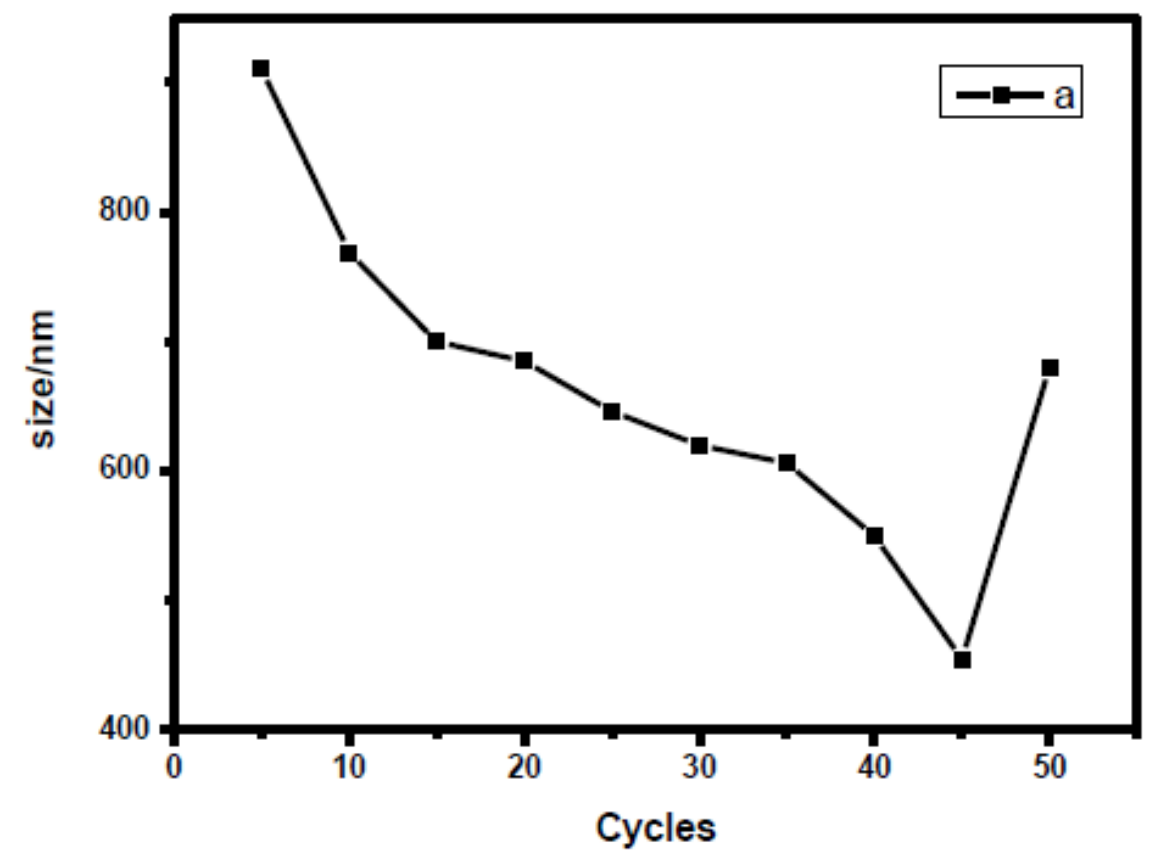




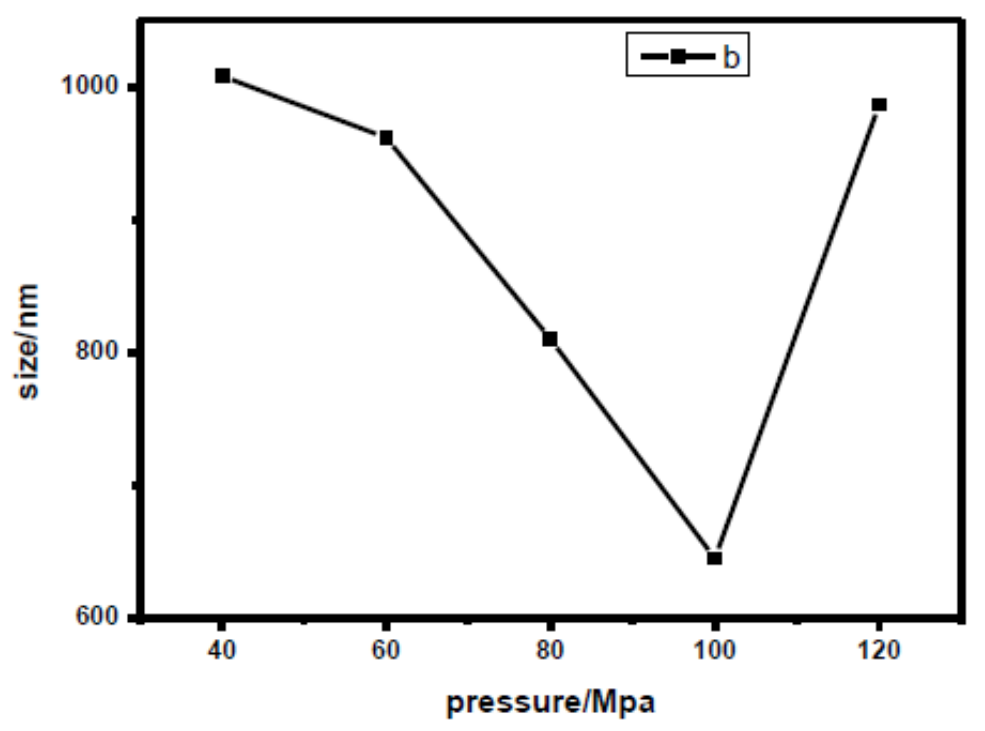

Fig. 1. Effects of homogeneous treatment on the particle size (a: Cycles; b: Pressure).

Effects of different homogeneous pressure on particle sizes are shown in Fig.1.b. The particle size of nanocellulose decreased with the increased homogeneous pressure until 100MPa, and then increased. It was due to the interaction of shearing force, collision force and cavitation interaction among the pineapple leaf cellulose. The molecular chain of nanocellulose was interrupted seriously and the particle size of nanocellulose decreased with the homogeneous pressure increased. But as the processing pressure continued to increase, the particle size of nanocellulose was prone to reunite by the van der Waals force, leading to the increased particle size.

Hence, the optimal homogeneous conditions were as follows: 3\% (w/w) pineapple leaf cellulose/BmimCl solution was through the high pressure homogenizer under an operating pressure of $100 \mathrm{MPa}$ with 45 cycles at room temperature.

\section{Structure Analysis of Nanocellulose by TEM, FT-IR, XRD, TGA}

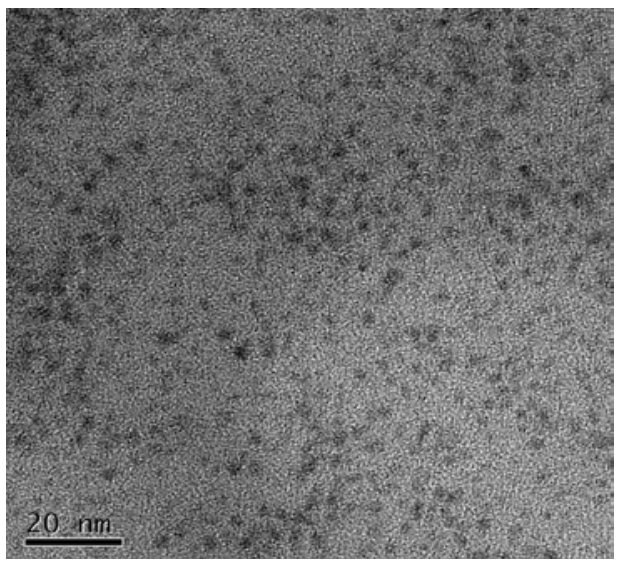

Fig. 2. TEM micrographs of nanocellulose.

The structure of pineapple leaf cellulose was destroyed during the process and the formed nanocellulose particles. After treatment under the optimal conditions, nanocellulose particles presented regular spherical shapes and good dispersion, with 4-10 diameter size shown in Fig. 2. 


\section{Infrared Spectrum Analysis}

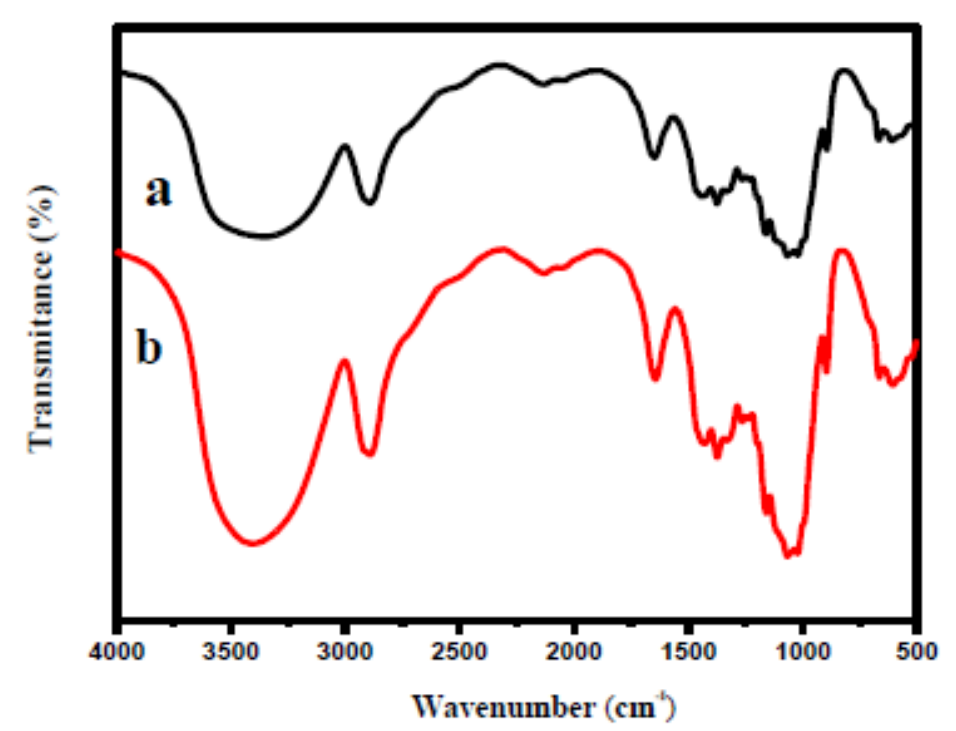

Fig. 3. FT-IR spectra of regenerated cellulose (a), nanocellulose (b).

Fig. 3 shows FT-IR spectra of regenerated cellulose (a), nanocellulose (b). The two spectra were very similar, which was demonstated that high pressure homogeneous process did not change the main structure of the pineapple leaf cellulose. The O-H vibration peak of nanocellulose was moved from to $3412 \mathrm{~cm}^{-1}$ to $3418 \mathrm{~cm}^{-1}$ during the process. It indicated that intermolecular and intramolecular hydrogen bonding between the cellulose molecular chains was receded.

Specially, the peak at $1429 \mathrm{~cm}^{-1}$ (CH2 scissor movement) was broaden after HPH, which was suggested the intramolecular hydrogen bonding of pineapple leaf cellulose (hydrogen bonding at C6) were destroyed during HPH pretreatment.

\section{Crystal Structure Analysis (XRD)}

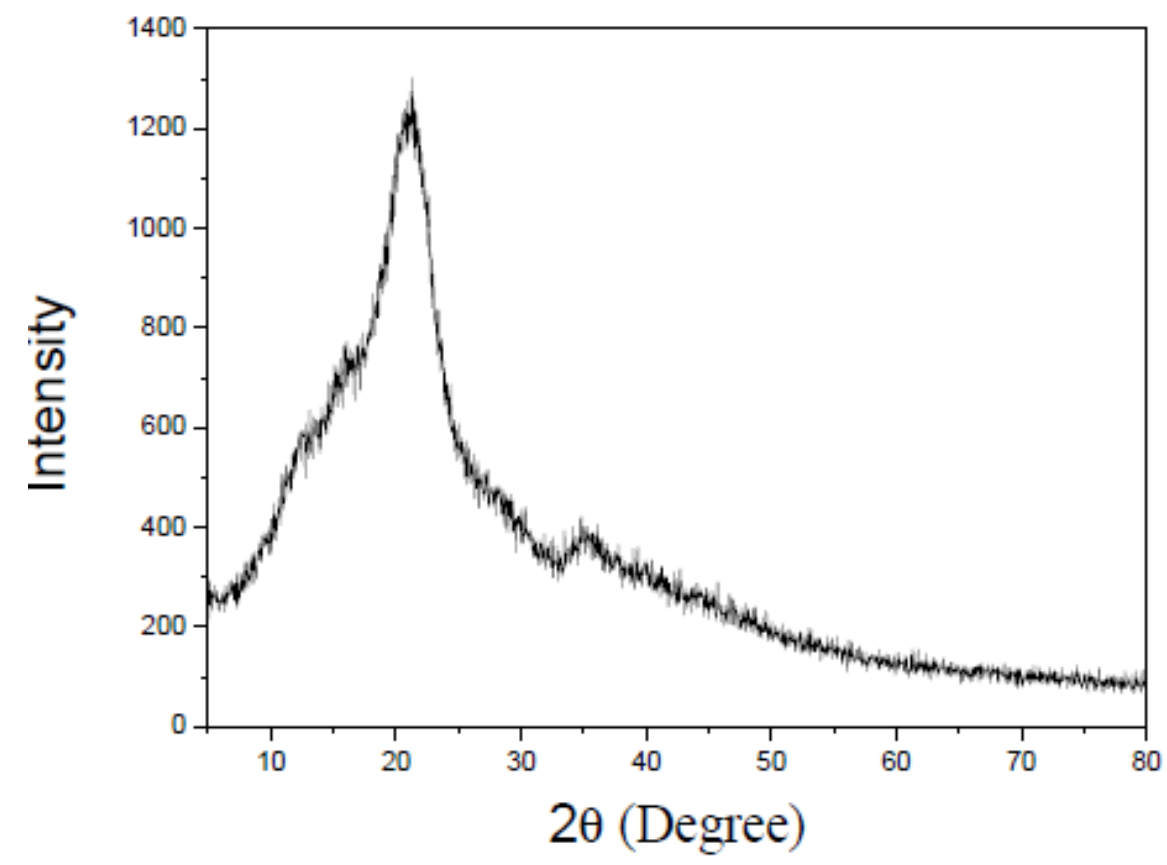

Fig. 4. XRD patterns of nanocellulose.

Fig. 4 shows the crystal structure of nanocellulose after HPH. It can be seen that nanocellulose has strong diffraction peak in $11.7^{\circ}, 20.3^{\circ}$ and $21.7^{\circ}$, which belonged to cellulose II structure $[15,16]$. 
Consistent with FT-IR results, high pressure homogeneous process is a physical process, and there is no obvious change in the structure of cellulose. However, the crystallinity was reduced from $69.68 \%$ to $34.25 \%$ after HPH treatment. This was due to intermolecular and intramolecular hydrogen bondings of the cellulose were attacked, and the ordered crystalline region changed to amorphous region, leading to the declined crystallinity dramatically [17].

\section{Thermo Gravimetric Analysis (TGA)}

The thermo gravimetric analysis of regenerated cellulose and nanocellulose are shown in Table 1 . The initial decomposition temperature and the termination decomposition temperature of pineapple leaf nanocellulose were down to $310.9{ }^{\circ} \mathrm{C}$ and $345.4{ }^{\circ} \mathrm{C}$ respectively. It suggested that the thermal stability decreased during HPH process, which was due to the damages in crystal region between cellulose.

Table 1. The different temperature of regenerated cellulose (a) and nanocellulose (b).

\begin{tabular}{llll}
\hline sample & initial temperature $/{ }^{\circ} \mathrm{C}$ & $\begin{array}{l}\text { Maximum decomposition } \\
\text { rate } /{ }^{\circ} \mathrm{C}\end{array}$ & $\begin{array}{l}\text { termination } \\
\text { temperature } /{ }^{\circ} \mathrm{C}\end{array}$ \\
\hline $\begin{array}{l}\text { Regenerated } \\
\text { cellulose }\end{array}$ & 312.6 & 340.6 & 354.2 \\
$\begin{array}{l}\text { Nanocellulose } \\
310.9\end{array}$ & 338.6 & 345.4 \\
\hline
\end{tabular}

\section{The Properties of Nanocellulose}

Table 2. The physical properties of regenerated cellulose and nanocellulose.

\begin{tabular}{lll}
\hline Item & regenerated cellulose & nanocellulose \\
\hline Specific surface area $\mathrm{m}^{2} / \mathrm{g}$ & 2.0 & 2.8 \\
porosity $\mathrm{cm}^{3} / \mathrm{g}$ & 2.5 & 2.1 \\
swelling capacity $\mathrm{ml} / \mathrm{g}$ & 4.0 & 1.7 \\
Water-Holding Capacity g/g & 4.9 & 5.4 \\
Oil-Holding Capacity ml/g & 5.6 & 5.8 \\
\hline
\end{tabular}

Table 2 presents the physical properties of regenerated cellulose and nanocellulose. It shows that the specific surface area increased and the porosity decreased after HPH. This was due to the cellulose reticular structure was destroyed in the process, with the large gap of mesh structure into a spherical particle, leading to increased the specific surface area and decreased the porosity [18].

Swelling capacity is a specific technical index for cellulose. Through the calculation of Eq.1, the swelling capacity of nanocellulose was down to $1.7 \mathrm{ml} / \mathrm{g}$ after $\mathrm{HPH}$. This was because the reticular structure of cellulose was destroyed from big mesh structure into round spherical particles. Therefore, reduced moisture was passed into the interior of the cellulose channels, thus affecting the swelling capacity of cellulose.

The water-holding capacity (whc) and oil-holding capacity (ohc) are reflecting the ability to absorb and retain water /oil of pineapple leaf cellulose. Table 2 shows the whc and ohc of nanocellulose were higher than regenerated cellulose. It was mainly because the specific surface area of cellulose increased and surface active was strengthened and enlarged the contact area with water molecules and grease molecules. Nanocellulose because of its good whc and ohc, so in make up cotton, nano mask has had the very big development, the partial products have industrialization, at the same time it also has application in cosmetics ingredients. 


\section{Summary}

In this study, the pineapple leaf nanocellulose was prepared by high pressure homogenization in ionic liquids. The effects of preparation process on particle sizes and structure were studied, and the optimal homogeneous conditions were $3 \%(\mathrm{w} / \mathrm{w})$ pineapple leaf cellulose/BmimCl solution through the high pressure homogenizer under an operating pressure $100 \mathrm{MPa}$ with 45 cycles at room temperature. The change of structure performance and the properties of pineapple leaf cellulose before and after HPH treatment was characterized. The results showed that nanocellulose particles are spherical and reunion with the diameter of 4-10 nm, and the crystal structure of nanocellulose belonged to cellulose II, but the crystallinity, thermal ability, porosity and swelling capacity were reduced. The specific surface area, water-holding capacity and oil-holding capacity of nanocellulose were increased after $\mathrm{HPH}$, which can be used for coating, film, textiles, cosmetics, food, composite materials, etc.

\section{Acknowledgement}

This research was financially supported by the Natural Science Foundation of Hainan Province (20155204) and the Fundamental Scientific Research Funds for Chinese Academy of Tropical Agricultural Sciences(1630062015020).

\section{References}

[1] M.A.S.A. Samir, F. Alloin, J.Y. Sachez, N.E. Kissi, A. Dufresne, Preparation of cellulose whiskers reinforced nanocomposites from an organic medium suspension, J. Macromolecules. 37 (2004) 1386-1393.

[2] R. Jung, H.S. Kim, Y. Kim, Electrically conductive transparent papers using multiwalled carbon nanotubes, J. Polym Sci. 46 (2008) 1235-1242.

[3] L.E. Millon, W.K. Wan, The polyvinyl alcohol-bacterial cellulose system as a new nanocomposite for biomedical applications, J. Biomed Mater Res B Appl Biomater. 79 (2006) 245-253.

[4] M. Murayama, M. Tabuchi, Development of UV-care cosmetic using Nata de Coco, J. Bio Ind. 24 (2007) 80-86.

[5] X.M. Li, H. Lan, Z.Z. Chen, The application of nanocrystalline cellulose in ice cream, J. Sci Tech Food Ind. 35 (2014) 294-299.

[6] S.S. Paes, S. Sun, W. Macnaughtan, R. Ibbett, J. Ganster, T.J. Foste, J.R. Mitchell ,The glass transition and crystallization of ball milled cellulose, J. Cellul. 17 (2010) 693-709.

[7] A. Mandal, D. Chakrabarty, Isolation of nanocellulose from waste sugarcane bagasse (SCB) and its characterization, J. Carbohydr Polym. 86 (2011) 1291-1299.

[8] J.I. Moran, V.A. Alvarez, V.P. Cyras, A. Vázquez, Extraction of cellulose and preparation of nanocellulose from sisal fibers, J. Cellul. 15 (2008) 149-159.

[9] W. Li, R. Wang, S.X. Liu, The preparation of nanocellulose, J. Prog Chem. 22 (2010) 2060-2070.

[10] J.H. Li, X.Y. Wei, Q.H. Wang, J.C. Chen, G. Chang, L.X. Kong, J.B. Su, Y.H. Liu, Homogeneous isolation of nanocellulose from sugarcane bagasse by high pressure homogenization, $\mathrm{J}$. Carbohydr Polym. 90 (2012) 1609-1613.

[11] T.k. Fu, J.H. Li, Q.H. Wang, H. Huang, X.Y. Wei, L.H. Cui, Y.H. Wang, F. Wang, Structure and Properties of Natural Cellulose Extracted from Pineapple Leaf, J. Adv Mater Res. 815 (2013) 379-385. 
[12] L. Kuniak, R.H. Marchessault, Study of the crosslinking reaction between epichlorohydrin and starch, J. Starch. 24 (1972) 110-116.

[13] A. Yolanda, M.E. Rosa, B. Vanesa, M. Esperanza, A.M.C. María , Starch, Functional Properties, and Microstructural Characteristics in Chickpea and Lentil As Affected by Thermal Processing, J. Agric Food Chem. 57 (2009) 10682-10688.

[14] F. Donsi, Y.W. Wang, J. Li, Preparation of curcumin submicrometer dispersions by high-pressure homogenization, J. Agric Food Chem. 58 (2010) 2848-2853.

[15] R.L. Wu, X.L. Wang, Y.Z. Wang, X.C. Bian, F. Li, Cellulose/soy protein isolate blend films prepared via room-temperature ionic liquid, J. Ind Eng Chem Res. 48 (2009) 7132-7136.

[16] P. Mansikkamaki, M. Lahtinen, K. Rissanen, Structural changes of cellulose crystallites induced by mercerisation in different solvent systems; determined by powder X-ray diffraction method, J. Cellul. 12 (2005) 233-242.

[17] D.S. Zhao, H. Li, J. Zhang, L.L. Fu, M.S. Liu, J.T. Fu, P.B. Ren, Dissolution of cellulose in phosphate-based ionic liquids, J. Carbohydr Polym. 87 (2012) 1490-1494.

[18] S. Houssine , Z. Qi , A.B. Lars , High-porosity aerogels of high specific surface area prepared from nanofibrillated cellulose (NFC), J. Compos Sci Technol. 71 (2011) 1593-1599. 